This item was submitted to Loughborough's Research Repository by the author.

Items in Figshare are protected by copyright, with all rights reserved, unless otherwise indicated.

\title{
Development of a scanning head for laser Doppler vibrometry (LDV) using dual optical wedges
}

PLEASE CITE THE PUBLISHED VERSION

http://dx.doi.org/10.1063/1.4845555

PUBLISHER

C) AIP Publishing

VERSION

VoR (Version of Record)

LICENCE

CC BY-NC-ND 4.0

\section{REPOSITORY RECORD}

Rothberg, Steve, and Mario Tirabassi. 2019. "Development of a Scanning Head for Laser Doppler Vibrometry (LDV) Using Dual Optical Wedges". figshare. https://hdl.handle.net/2134/14047. 


\section{AD| $\begin{aligned} & \text { Review of } \\ & \text { Scientific Instruments }\end{aligned}$}

Development of a scanning head for laser Doppler vibrometry (LDV) using dual optical wedges

S. J. Rothberg and M. Tirabassi

Citation: Review of Scientific Instruments 84, 121704 (2013); doi: 10.1063/1.4845555

View online: http://dx.doi.org/10.1063/1.4845555

View Table of Contents: http://scitation.aip.org/content/aip/journal/rsi/84/12?ver=pdfcov

Published by the AIP Publishing

The Complete Guide to Building an Instrument Control System

» Learn more at www.NI.com/Instrument-Control

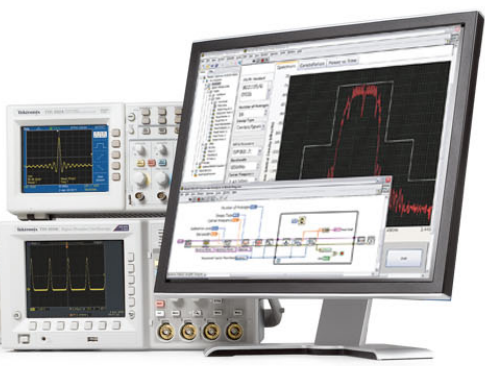




\title{
Development of a scanning head for laser Doppler vibrometry (LDV) using dual optical wedges
}

\author{
S. J. Rothberga) and M. Tirabassib) \\ Wolfson School of Mechanical and Manufacturing Engineering, Loughborough University, Loughborough, \\ Leicestershire LE11 3TU, United Kingdom
}

(Received 9 June 2013; accepted 6 October 2013; published online 18 December 2013)

\begin{abstract}
A new laser Doppler vibrometry scanning head is proposed based on a pair of rotating optical wedges. A comprehensive mathematical model is developed and used to demonstrate how the wedges can be configured to scan point-by-point, in a line, in a circle, and in a combination of the two such that a straight line scan could be performed on a structure during rotation. Inevitable misalignments are incorporated into the model and considered quantitatively for the circular tracking application. Certain advantages are apparent over systems based on dual mirrors and a Dove prism in terms of lower apparent velocities at low rotation orders. A scanning head design for the circular tracking application is presented, together with experimental data showing good agreement between predicted and measured apparent velocities caused by misalignments. (C) 2013 AIP Publishing LLC. [http://dx.doi.org/10.1063/1.4845555]
\end{abstract}

\section{INTRODUCTION}

The laser vibrometer is now well established as an effective, non-contact alternative to the use of traditional vibration transducers. Special benefits accrue when certain measurement constraints are imposed, for example, by the context, which may demand high frequency operation, high spatial resolution or remote transducer operation, or by the structure itself, which may be hot, light, or rotating. Among its attractions is the ease with which both the direction of the laser beam and its incident point can be manipulated, as recognised at an early stage with the introduction of the Scanning Laser Doppler Vibrometer (SLDV), ${ }^{1}$ scanning point-bypoint across a structure with particular applications in the automotive $^{2-4}$ and aerospace ${ }^{5}$ industries. Today's commercial state-of-the-art offers automated, tri-axial vibration surveys on large, three-dimensional structures (such as a vehicle) using three SLDVs each mounted on a robot arm. ${ }^{6}$

In addition to this point-by-point operation, it is possible to configure a SLDV to function in a continuous scanning mode, initially enabling extraction of particular vibration components $^{7}$ and ultimately enabling the target velocity profile along a pre-determined path to be determined in a single measurement. In this latter case, post-processing of the measured velocity results in a series of coefficients that describe the operational deflection shape (ODS) or the mode shape.$^{8-14}$ If the scan frequency is synchronised with the target motion frequency, it is possible to perform a tracking laser vibrometer measurement in which the probe laser beam remains fixed on a particular point on the target. ${ }^{15}$ Tracking measurements have been performed on a number of rotor applications, ${ }^{15-17}$ as well as on belts (partial track) ${ }^{18}$ and on targets with oscillating parts fixed to a component with a large whole body motion such as windscreen wipers. ${ }^{19}$ On rotating structures,

\footnotetext{
a) Author to whom correspondence should be addressed. Electronic mail: s.j.rothberg@lboro.ac.uk

b) Present address: Bruel and Kjaer VTS Limited, Herts, United Kingdom.
}

the attractions of tracking and scanning simultaneously have also been explored. ${ }^{20,21}$

By far the most popular means by which to manipulate the probe laser beam is by using a pair of orthogonally mounted galvanometer mirrors. This approach works well for point-by-point relocation but the inertia of the mirrors, whose accelerations must reach high levels to sustain the required amplitudes of oscillatory motion at high frequency, can limit performance for continuous duty. To overcome this problem in applications such as high speed tracking of rotors, self-tracking systems have been introduced using a combination of a mirror fixed to the rotor itself and a remote mirror that is stationary. ${ }^{22,23}$ This arrangement can be supplemented by scanning (with a traditional dual mirror system) onto the mirror fixed to the rotor to perform scanning while tracking. ${ }^{24}$ An alternative rotor tracking system based on a Dove prism has recently been proposed ${ }^{25}$ and is now available commercially. ${ }^{26}$ The Dove prism rotates continuously in the tracking system, rather than in the oscillatory fashion of scanning mirrors, and offers the additional advantage that it need only rotate at half the speed of its target to maintain track.

This paper explores the use of a pair of optical wedges as a scanning head and reports initial development of an experimental system. Like the Dove prism SLDV, this dual wedge SLDV would overcome the limitation imposed by the inertia of oscillatory mirrors. In a rotor tracking system, the wedges would rotate continuously, only requiring acceleration sufficient to match the structure under test. The wedges cannot match the additional advantage of the Dove prism (half speed rotation) but they could offer much of the versatility of the scanning mirrors in terms of point-by-point relocation and the scanning of paths other than circular. ${ }^{27,28}$ Both Dove prism and wedges require balancing.

Detailed study of proposed new systems and correspondingly detailed comparison with existing systems has been made possible by the recent introduction of a universal framework for predicting measured velocities, beam paths, and scan 
paths in laser Doppler vibrometry (LDV) systems, with full geometrical accuracy including routine incorporation of the small but inevitable misalignments to be found in all real systems. ${ }^{29}$ The framework also acknowledges the relatively neglected effect of Doppler shifts occurring when laser beam deflection (reflection and refraction) occurs at moving optical devices. Section II sets out how measured and surface velocities are related to each other in general terms. This is then followed by modelling of the dual wedge SLDV within this framework. While focussed on the analysis of a specific system, the model is set out in a manner that is intended to emphasise the generic nature of the modelling framework.

\section{MEASURED VELOCITY AND SURFACE VELOCITY}

The fundamental relationship between measured velocity, $U_{m}$, and surface velocity at a point $P^{\prime}, \overrightarrow{V_{P^{\prime}}}$, is captured in the expression: ${ }^{30}$

$$
U_{m}=\frac{1}{2}\left(\hat{b}_{n+1}-\hat{b}_{n}\right) \cdot \overrightarrow{V_{P^{\prime}}}
$$

in which $\hat{b}_{n+1}$ and $\hat{b}_{n}$ are, respectively, unit vectors for the laser beam directions immediately after and immediately before the point $P^{\prime}$. Equation (1) can be applied to incidence at a target surface and to deflections at optical devices. For scattering from the target with light collected in direct backscatter, $\hat{b}_{n+1}=-\hat{b}_{n}$ and Eq. (1) simplifies to

$$
U_{m}=-\hat{b}_{n} \cdot \overrightarrow{V_{P^{\prime}}}
$$

With light collected in direct backscatter, deflections at optical devices occur at the same point in both the outgoing and incoming beam path. Based on Eq. (1), the measured velocity associated with the double pass through a deflection point $P^{\prime}$ on an optical device is therefore given by

$$
\begin{aligned}
\mathrm{U}_{\mathrm{m}} & =\frac{1}{2}\left(\hat{\mathrm{b}}_{\mathrm{n}+1}-\hat{\mathrm{b}}_{\mathrm{n}}\right) \cdot \overrightarrow{\mathrm{V}_{\mathrm{P}^{\prime}}}+\frac{1}{2}\left(-\hat{\mathrm{b}}_{\mathrm{n}}--\hat{\mathrm{b}}_{\mathrm{n}+1}\right) \cdot \overrightarrow{\mathrm{V}_{\mathrm{P}^{\prime}}} \\
& =\left(\hat{\mathrm{b}}_{\mathrm{n}+1}-\hat{\mathrm{b}}_{\mathrm{n}}\right) \cdot \overrightarrow{\mathrm{V}_{\mathrm{P}^{\prime}}},
\end{aligned}
$$

where the unit vector subscripts refer to the outgoing beam path. The total measured velocity is given by the sum of the individual velocities associated with the Doppler shifts at deflections at optical devices and at the target.

Equations (2a) and (2b) highlight the attraction of vector descriptions for surface velocity and beam orientation. This is facilitated by definition of a global $x y z$ coordinate system with origin $O$ fixed in space and unit vectors $\hat{x}, \hat{y}$, and $\hat{z}$ associated with its axes. Considering the general case of a rotating and vibrating target, $O$ coincides with a convenient reference point on the target rotation axis in the absence of any vibration. The $z$-axis is aligned with the target rotation axis, again in the absence of vibration. With vibration, this target reference point moves to $O^{*}$ as a consequence of its velocity $\overrightarrow{V_{O^{*}}}$. From fundamental kinematics, surface velocity at the incident point $P^{\prime}$ is then conveniently written in terms of the sum of the velocity of the reference point and the velocity of $P^{\prime}$ relative to $O^{*}$ as a result of rotation at angular velocity $\vec{\omega}$ about an instantaneous rotation axis passing through $O^{*}$ :

$$
\overrightarrow{V_{P^{\prime}}}=\overrightarrow{V_{O^{*}}}+\left(\vec{\omega} \times \overrightarrow{r_{P^{\prime}} / O^{*}}\right)
$$

where $\overrightarrow{r_{P^{\prime} / O^{*}}}$ is the position vector for $P^{\prime}$ relative to $O^{*} . \vec{\omega}$ includes both continuous target rotation and angular oscillations around the three coordinate axes. For vibrating targets, $P^{\prime}$ can change continuously and can also be affected by target shape but, for an illuminated target element considered to be rigid, Eq. (2a) can be expanded in terms of any known point along the line of the laser beam, ${ }^{31}$ described by the position vector $\overrightarrow{r_{0}}$ :

$$
U_{m}=-\hat{b}_{n} \cdot \overrightarrow{V_{P^{\prime}}}=-\hat{b}_{n} \cdot\left(\overrightarrow{V_{O^{*}}}+\vec{\omega} \times\left[\overrightarrow{r_{0}}-\overrightarrow{O O^{*}}\right]\right),
$$

where $\overrightarrow{\mathrm{OO}^{*}}$ is the target displacement vector associated with the velocity $\overrightarrow{\mathrm{V}_{\mathrm{O}^{*}}}$. For applications such as the tracking of a bladed disc, the relative vibration velocity associated with target flexibility must be added to the rigid element velocity. ${ }^{32}$ This must be written to accommodate time-dependency in the illuminated point, modifying Eq. (4a) to become

$$
\begin{aligned}
U_{m} & =-\hat{b}_{n} \cdot \overrightarrow{V_{P^{\prime}(t)}} \\
& =-\hat{b}_{n} \cdot\left(\overrightarrow{V_{O^{*}}}+\vec{\omega} \times\left[\overrightarrow{r_{0}}-\overrightarrow{O O^{*}}\right]+\overrightarrow{V_{f}\left(P^{\prime}(t)\right)}\right),
\end{aligned}
$$

where $\overrightarrow{V_{f}\left(P^{\prime}(t)\right)}$ is the vector velocity at point $P^{\prime}(t)$ associated with target flexibility. Later simulations restrict target motions to an in-plane $(x)$ whole body vibration, $V_{O^{*} x} \hat{x}$, and an out-of plane (z) flexible vibration, $V_{f z}\left(P^{\prime}(t)\right) \hat{z}$, combined with target rotation at $\Omega_{\mathrm{T}}$ around $\hat{z}$. This represents the important case of a rotating bladed disc attached to a whirling shaft for which the measured velocity, in terms of point $T^{\prime}$ (where the laser beam intersects the $x y$ plane in which $O$ is located) along the beam, is written

$$
\begin{aligned}
U_{m}= & -\hat{b}_{n} \cdot\left(V_{O^{*} x} \hat{x}+\Omega_{T} \hat{z}\right. \\
& \left.\times\left[\overrightarrow{O T^{\prime}}-\overrightarrow{O O^{*}}\right]+V_{f z}\left(P^{\prime}(t)\right) \hat{z}\right) .
\end{aligned}
$$

In applying the same principles to an optical device, a particular labelling convention is adopted. Without misalignment, a significant point such as a reference point on a rotation axis might be identified as $P$. With misalignments added, the new position of this point will be labelled $P^{*}$ and point $P$ may no longer lie on a surface of the optical device. On the surface containing $P^{*}$, the point through which the laser beam actually passes will be labelled $P^{\prime}$ (see Figures 1(a) and 1(b)). Vectors $\overrightarrow{O P}, \overrightarrow{O P^{*}}$, and $\overrightarrow{O P^{\prime}}$ define these positions while, for example, the vector $\overrightarrow{P^{*} P^{\prime}}$ defines the path from $P^{*}$ to $P^{\prime} . \overrightarrow{O P}$ and $\overrightarrow{O P^{*}}$ will be inputs to the models while $\overrightarrow{O P^{\prime}}$ and $\overrightarrow{P^{*} P^{\prime}}$ will be found as part of the model. Adopting this convention and combining Eqs. (2b) and (3), the measured velocity associated with deflection at an optical device can be written

$$
U_{m}=\left(\hat{b}_{n+1}-\hat{b}_{n}\right) \cdot\left(\overrightarrow{V_{P^{*}}}+\vec{\omega} \times \overrightarrow{P^{*} P^{\prime}}\right) \text {. }
$$

If $P^{*}$ is a point on the device rotation axis, $\overrightarrow{V_{P^{*}}}$ will usually be zero, simplifying Eq. (5). Exceptions include modelling the effects of vibrations of the device itself. 


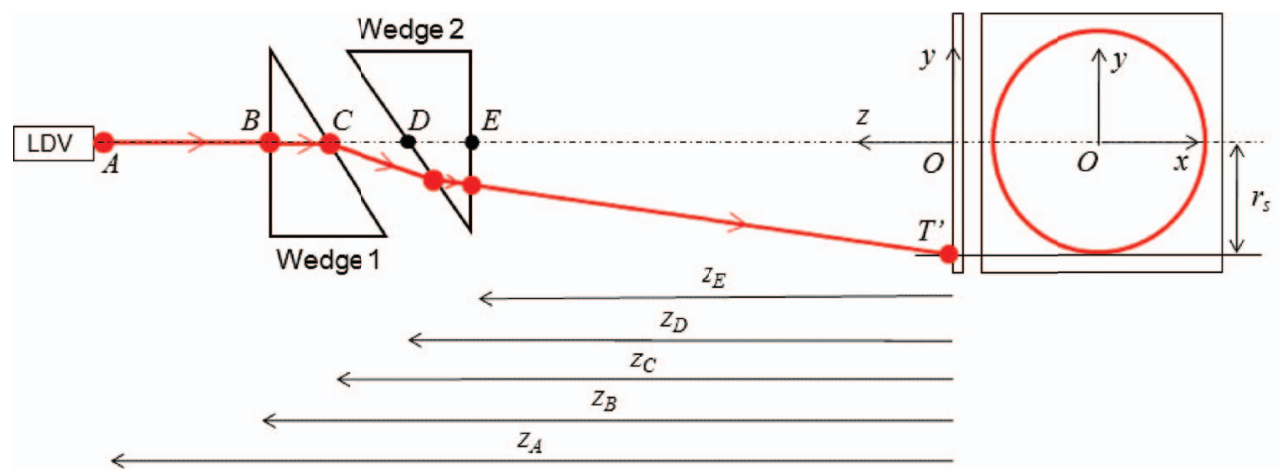

(a)

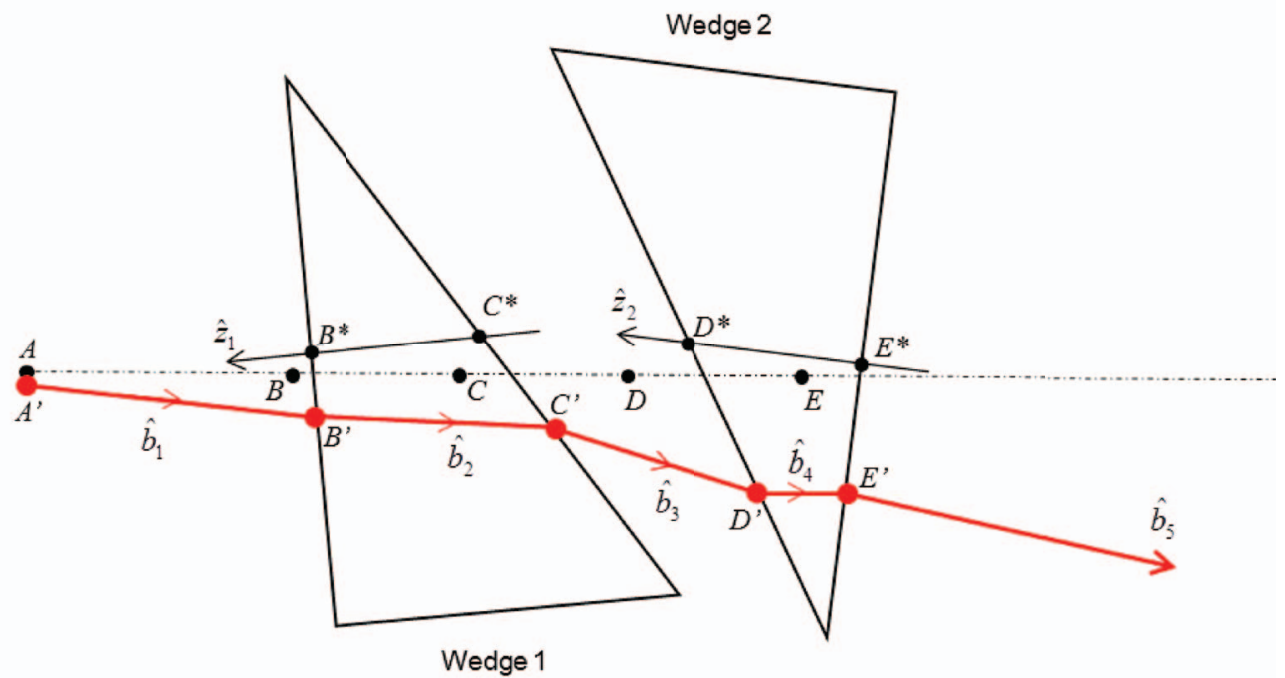

(b)

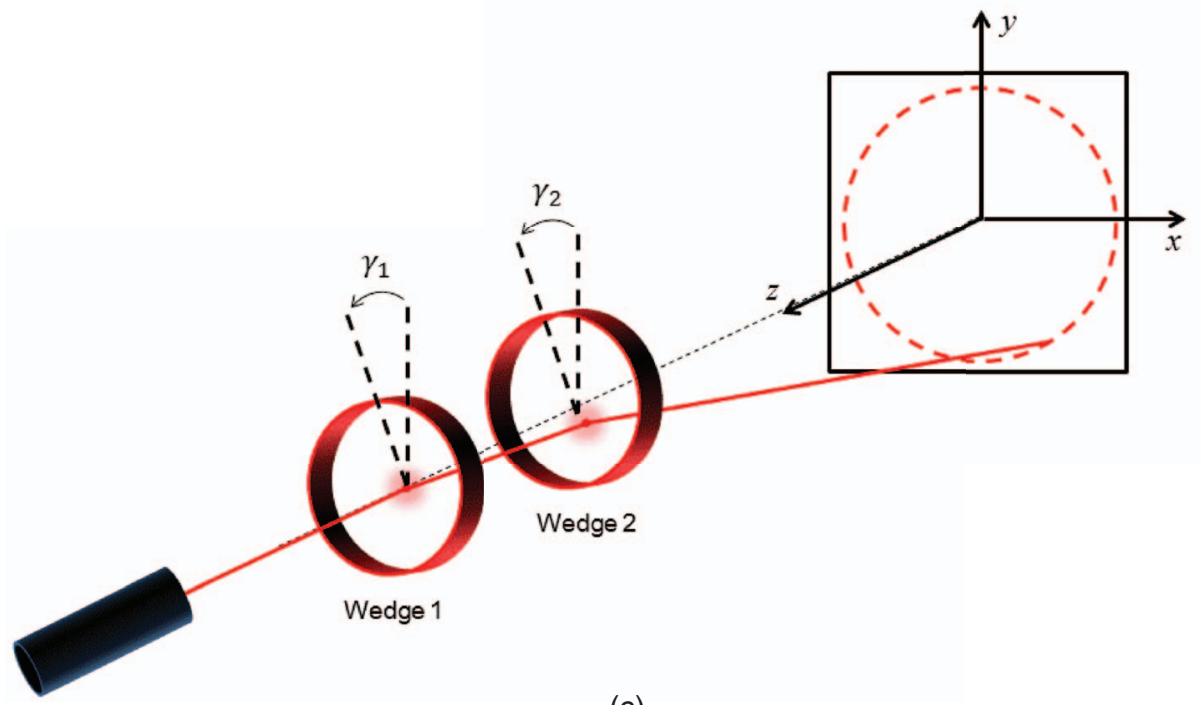

(c)

FIG. 1. (a) Dual wedge SLDV without misalignments, showing a scanned circle. Reprinted with permission from S. J. Rothberg and M. Tirabassi, AIP Conf. Proc. 1457, 5 (2012). Copyright 2012 American Institute of Physics. ${ }^{34}$ (b) Misalignment of the wedges and laser head in the dual wedge SLDV. Reprinted with permission from S. J. Rothberg and M. Tirabassi, AIP Conf. Proc. 1457, 5 (2012). Copyright 2012 American Institute of Physics. ${ }^{34}$ (c) Rotation of the wedges around z-axis.

\section{DUAL WEDGE SLDV: THEORETICAL INVESTIGATION}

The novel dual wedge SLDV is shown in Figure 1(a). In a system without misalignments, it is possible to define the points A to E. Point A corresponds to the nominal position of the laser source, points $\mathrm{B}$ and $\mathrm{C}$ define the rotation axis of the first wedge while points $\mathrm{D}$ and $\mathrm{E}$ define the rotation axis for the second wedge. 
Points $\mathrm{A}$ and $\mathrm{B}$ are written in vector form as

$$
\begin{aligned}
& \overrightarrow{O A}=\left[\begin{array}{lll}
\hat{x} & \hat{y} & \hat{z}
\end{array}\right]\left[\begin{array}{lll}
0 & 0 & z_{A}
\end{array}\right]^{T}, \\
& \overrightarrow{O B}=\left[\begin{array}{lll}
\hat{x} & \hat{y} & \hat{z}
\end{array}\right]\left[\begin{array}{lll}
0 & 0 & z_{B}
\end{array}\right]^{T} .
\end{aligned}
$$

Figure 1(b) shows the wedges with translational and angular misalignments present, including the points $A^{\prime}, B^{*}, C^{*}, D^{*}$, and $E^{*}$. The following equation show how translational misalignments are incorporated into the analysis. The modified vectors $\overrightarrow{O A^{\prime}}$ and $\overrightarrow{O B^{*}}$ are written as

$$
\begin{aligned}
& \overrightarrow{O A^{\prime}}=\overrightarrow{O A}+\left[\begin{array}{lll}
\hat{x} & \hat{y} & \hat{z}
\end{array}\right]\left[\begin{array}{lll}
\Delta x_{A} & \Delta y_{A} & \Delta z_{A}
\end{array}\right]^{T}, \\
& \overrightarrow{O B^{*}}=\overrightarrow{O B}+\left[\begin{array}{lll}
\hat{x} & \hat{y} & \hat{z}
\end{array}\right]\left[\begin{array}{lll}
\Delta x_{B} & \Delta y_{B} & \Delta z_{B}
\end{array}\right]^{T} .
\end{aligned}
$$

Points D and $\mathrm{D}^{*}$ on the second wedge are written in a similar fashion:

$$
\begin{gathered}
\overrightarrow{O D}=\left[\begin{array}{lll}
\hat{x} & \hat{y} & \hat{z}
\end{array}\right]\left[\begin{array}{lll}
0 & 0 & z_{D}
\end{array}\right]^{T}, \\
\overrightarrow{O D^{*}}=\overrightarrow{O D}+\left[\begin{array}{lll}
\hat{x} & \hat{y} & \hat{z}
\end{array}\right]\left[\begin{array}{ll}
\Delta x_{D} \Delta y_{D} \Delta z_{D}
\end{array}\right]^{T} .
\end{gathered}
$$

Expressions for points $C^{*}$ and $E^{*}$ are defined in terms of $B^{*}$ and $D^{*}$, respectively, and are written in terms of the wedge rotation axes which are themselves affected by angular misalignments. These equations for the wedge rotation axis vectors exemplify the use of rotation matrices (see the Appendix) to modify an initial orientation (in this case $\hat{z}$ ) to accommodate angular misalignments within the modelling framework:

$$
\begin{aligned}
& \hat{z}_{1}=\left[\begin{array}{lll}
\hat{x} & \hat{y} & \hat{z}
\end{array}\right]\left[x, \alpha_{1}\right]\left[\begin{array}{ll}
y, \beta_{1}
\end{array}\right]\left[\begin{array}{lll}
0 & 0 & 1
\end{array}\right]^{T}, \\
& \hat{z}_{2}=\left[\begin{array}{lll}
\hat{x} & \hat{y} & \hat{z}
\end{array}\right]\left[x, \alpha_{2}\right]\left[\begin{array}{ll}
y, \beta_{2}
\end{array}\right]\left[\begin{array}{lll}
0 & 0 & 1
\end{array}\right]^{T},
\end{aligned}
$$

from which points $C^{*}$ and $E^{*}$ are written as

$$
\begin{aligned}
& \overrightarrow{O C^{*}}=\overrightarrow{O B^{*}}-|\overrightarrow{B C}| \hat{z}_{1}, \\
& \overrightarrow{O E^{*}}=\overrightarrow{O D^{*}}-|\overrightarrow{D E}| \hat{z}_{2} .
\end{aligned}
$$

The beam path is obviously affected by refractions at each of the four interfaces, requiring knowledge of the corresponding surface normals. These are written in terms of initial alignments modified by a series of rotation matrices. The first rotation matrices are for wedge angle $\psi$ with a first subscript " 1 " and " 2 " denoting the wedge and second subscripts " 1 " and " 2 " indicating the first or second face of each wedge on the outward beam path. The relative orientation of the wedges shown in Figures 1(a) and 1(b) is arbitrary. The second rotation matrix describes rotations around the z-axis by angles $\gamma_{1}$ and $\gamma_{2}$ for each wedge, respectively, which combine initial angular position with any additional discrete or continuous rotation. These rotations are shown in Figure 1(c). Finally, rotation matrices are used to include angular misalignments in the same way as in Eqs. (9a) and (9b),

$$
\begin{aligned}
& \hat{n}_{B^{*}}=\left[\begin{array}{lll}
\hat{x} & \hat{y} & \hat{z}
\end{array}\right]\left[x, \alpha_{1}\right]\left[y, \beta_{1}\right]\left[z, \gamma_{1}\right]\left[x, \psi_{11}\right]\left[\begin{array}{lll}
0 & 0 & 1
\end{array}\right]^{T}, \\
& \hat{n}_{C^{*}}=\left[\begin{array}{lll}
\hat{x} & \hat{y} & \hat{z}
\end{array}\right]\left[x, \alpha_{1}\right]\left[y, \beta_{1}\right]\left[z, \gamma_{1}\right]\left[x, \psi_{12}\right]\left[\begin{array}{lll}
0 & 0 & 1
\end{array}\right]^{T},
\end{aligned}
$$

$$
\hat{n}_{D^{*}}=\left[\begin{array}{lll}
\hat{x} & \hat{y} & z
\end{array}\right]\left[x, \alpha_{2}\right]\left[y, \beta_{2}\right]\left[z, \gamma_{2}\right]\left[x, \psi_{21}\right]\left[\begin{array}{lll}
0 & 0 & 1
\end{array}\right]^{T},
$$

$$
\hat{n}_{E^{*}}=\left[\begin{array}{lll}
\hat{x} & \hat{y} & \hat{z}
\end{array}\right]\left[x, \alpha_{2}\right]\left[y, \beta_{2}\right]\left[z, \gamma_{2}\right]\left[x, \psi_{22}\right]\left[\begin{array}{lll}
0 & 0 & 1
\end{array}\right]^{T} .
$$

Initial beam orientation also makes use of rotation matrices to accommodate angular misalignment and is written

$$
\hat{b}_{1}=\left[\begin{array}{lll}
\hat{x} & \hat{y} & \hat{z}
\end{array}\right]\left[x, \alpha_{L}\right]\left[y, \beta_{L}\right]\left[\begin{array}{lll}
0 & 0 & -1
\end{array}\right]^{T} .
$$

Orientations following each refraction are derived using vector expressions ${ }^{33}$ that require the vector expressions for each surface normal in Eqs. (11a)-(11d):

$$
\begin{aligned}
\hat{b}_{2}= & \left(\hat{b}_{1}-\left(\hat{b}_{1} \cdot \hat{n}_{B^{*}}\right) \hat{n}_{B^{*}}\right) \frac{\varepsilon_{0}}{\varepsilon_{1}} \\
& -\left(\sqrt{1-\left(\frac{\varepsilon_{0}}{\varepsilon_{1}}\right)^{2}\left(1-\left(\hat{b}_{1} \cdot \hat{n}_{B^{*}}\right)^{2}\right)}\right) \hat{n}_{B^{*}}, \\
\hat{b}_{3}= & \left(\hat{b}_{2}-\left(\hat{b}_{2} \cdot \hat{n}_{C^{*}}\right) \hat{n}_{C^{*}}\right) \frac{\varepsilon_{1}}{\varepsilon_{0}} \\
& -\left(\sqrt{1-\left(\frac{\varepsilon_{1}}{\varepsilon_{0}}\right)^{2}\left(1-\left(\hat{b}_{2} \cdot \hat{n}_{C^{*}}\right)^{2}\right)}\right) \hat{n}_{C^{*}}, \\
\hat{b}_{4}= & \left(\hat{b}_{3}-\left(\hat{b}_{3} \cdot \hat{n}_{D^{*}}\right) \hat{n}_{D^{*}}\right) \frac{\varepsilon_{0}}{\varepsilon_{2}} \\
& -\left(\sqrt{1-\left(\frac{\varepsilon_{0}}{\varepsilon_{2}}\right)^{2}\left(1-\left(\hat{b}_{3} \cdot \hat{n}_{D^{*}}\right)^{2}\right)}\right) \hat{n}_{D^{*},}, \\
\hat{b}_{5}= & \left(\hat{b}_{4}-\left(\hat{b}_{4} \cdot \hat{n}_{E^{*}}\right) \hat{n}_{E^{*}} \frac{\varepsilon_{2}}{\varepsilon_{0}}\right. \\
& -\left(\sqrt{1-\left(\frac{\varepsilon_{2}}{\varepsilon_{0}}\right)^{2}\left(1-\left(\hat{b}_{4} \cdot \hat{n}_{E^{*}}\right)^{2}\right)}\right) \hat{n}_{E^{*} .} . \\
& \left(\sqrt{1-{ }^{2}}\right)
\end{aligned}
$$

Attention is now turned to the identification of deflection points which define beam path and enable derivation of the surface velocities that lead to measured velocity. Key points of incidence are found sequentially beginning from the position of the laser source. A system of three equations is solved; the first is a vector triangle relating the unknown point of incidence to the previous deflection point (or laser source position) through knowledge of beam orientation, the second is a vector triangle relating the unknown point of incidence to a known point on the same optical surface, and the third is a dot product between the surface normal and a vector in the plane of the surface. Following this pattern, incidence points $B^{\prime}, C^{\prime}, D^{\prime}$, and $E^{\prime}$ (all shown in Figure 1(b)) are obtained as follows:

$$
\begin{gathered}
\overrightarrow{O B^{\prime}}=\overrightarrow{O A^{\prime}}+\left|\overrightarrow{A^{\prime} B^{\prime}}\right| \hat{b}_{1}, \\
\overrightarrow{O B^{\prime}}=\overrightarrow{O B^{*}}+\overrightarrow{B^{*} B^{\prime}}, \\
\overrightarrow{B^{*} B^{\prime}} \cdot \hat{n}_{B^{*}}=0,
\end{gathered}
$$


leading to

$$
\begin{gathered}
\overrightarrow{O B^{\prime}}=\overrightarrow{O A^{\prime}}+\left[\frac{\left(\overrightarrow{O B^{*}}-\overrightarrow{O A^{\prime}}\right) \cdot \hat{n}_{B^{*}}}{\hat{b}_{1} \cdot \hat{n}_{B^{*}}}\right] \hat{b}_{1}, \\
\overrightarrow{O C^{\prime}}=\overrightarrow{O B^{\prime}}+\left|\overrightarrow{B^{\prime} C^{\prime}}\right| \hat{b}_{2}, \\
\overrightarrow{O C^{\prime}}=\overrightarrow{O C^{*}}+\overrightarrow{C^{*} C^{\prime}}, \\
\overrightarrow{C^{*} C^{\prime}} \cdot \hat{n}_{C^{*}}=0,
\end{gathered}
$$

leading to

$$
\begin{aligned}
& \overrightarrow{O C^{\prime}}=\overrightarrow{O B^{\prime}}+\left[\frac{\left(\overrightarrow{O C^{*}}-\overrightarrow{O B^{\prime}}\right) \cdot \hat{n}_{C^{*}}}{\hat{b}_{2} \cdot \hat{n}_{C^{*}}}\right] \hat{b}_{2}, \\
& \overrightarrow{O D^{\prime}}=\overrightarrow{O C^{\prime}}+\left|\overrightarrow{C^{\prime} D^{\prime}}\right| \hat{b}_{3}, \\
& \overrightarrow{O D^{\prime}}=\overrightarrow{O D^{*}}+\overrightarrow{D^{*} D^{\prime}}, \\
& \overrightarrow{D^{*} D^{\prime}} \cdot \hat{n}_{D^{*}}=0,
\end{aligned}
$$

leading to

$$
\begin{gathered}
\overrightarrow{O D^{\prime}}=\overrightarrow{O C^{\prime}}+\left[\frac{\left(\overrightarrow{O D^{*}}-\overrightarrow{O C^{\prime}}\right) \cdot \hat{n}_{D^{*}}}{\hat{b}_{3} \cdot \hat{n}_{D^{*}}}\right] \hat{b}_{3}, \\
\overrightarrow{O E^{\prime}}=\overrightarrow{O D^{\prime}}+\left|\overrightarrow{D^{\prime} E^{\prime}}\right| \hat{b}_{4}, \\
\overrightarrow{O E^{\prime}}=\overrightarrow{O E^{*}}+\overrightarrow{E^{*} E^{\prime}}, \\
\overrightarrow{E^{*} E^{\prime}} \cdot \hat{n}_{E^{*}}=0,
\end{gathered}
$$

leading to

$$
\overrightarrow{O E^{\prime}}=\overrightarrow{O D^{\prime}}+\left[\frac{\left(\overrightarrow{O E^{*}}-\overrightarrow{O D^{\prime}}\right) \cdot \hat{n}_{E^{*}}}{\hat{b}_{4} \cdot \hat{n}_{E^{*}}}\right] \hat{b}_{4} .
$$

The point $\mathrm{T}^{\prime}$ where the laser beam intersects the $x y$ plane is found in similar but slightly simpler fashion

$$
\begin{gathered}
\overrightarrow{O T^{\prime}}=\overrightarrow{O E^{\prime}}+\left|\overrightarrow{E^{\prime} T^{\prime}}\right| \hat{b}_{5}, \\
\overrightarrow{O T^{\prime}} \cdot \hat{z}=0,
\end{gathered}
$$

leading to

$$
\overrightarrow{O T^{\prime}}=\overrightarrow{O E^{\prime}}-\left[\frac{\overrightarrow{O E^{\prime}} \cdot \hat{z}}{\hat{b}_{5} \cdot \hat{z}}\right] \hat{b}_{5}
$$

Total measured velocity is the sum of the measured velocities from points $B^{\prime}, C^{\prime}, D^{\prime}, E^{\prime}$, and $T^{\prime}$ and is written as

$$
\begin{aligned}
U_{m}= & \left(\hat{b}_{2}-\hat{b}_{1}\right) \cdot \overrightarrow{V_{B^{\prime}}}+\left(\hat{b}_{3}-\hat{b}_{2}\right) \cdot \overrightarrow{V_{C^{\prime}}}+\left(\hat{b}_{4}-\hat{b}_{3}\right) \cdot \overrightarrow{V_{D^{\prime}}} \\
& +\left(\hat{b}_{5}-\hat{b}_{4}\right) \cdot \overrightarrow{V_{E^{\prime}}}-\hat{b}_{3} \cdot \overrightarrow{V_{T^{\prime}}} .
\end{aligned}
$$

The surface velocities at the deflection points on each wedge follow the form of Eq. (5):

$$
\begin{aligned}
& \overrightarrow{V_{B^{\prime}}}=\dot{\gamma}_{1} \hat{z}_{1} \times \overrightarrow{B^{*} B^{\prime}}, \\
& \overrightarrow{V_{C^{\prime}}}=\dot{\gamma}_{1} \hat{z}_{1} \times \overrightarrow{C^{*} C^{\prime}}, \\
& \overrightarrow{V_{D^{\prime}}}=\dot{\gamma}_{2} \hat{z}_{2} \times \overrightarrow{D^{*} D^{\prime}},
\end{aligned}
$$

$$
\overrightarrow{V_{E^{\prime}}}=\dot{\gamma}_{2} \hat{z}_{2} \times \overrightarrow{E^{*} E^{\prime}}
$$

while the relevant component of the target surface velocity is written according to Eq. (4b).

As presented to this point, the model is totally general. The particular path scanned depends on the functions used for $\gamma_{1}$ and $\gamma_{2}$, and this flexibility delivers the versatility of the proposed dual wedge SLDV.

\section{A. Point-by-point and line scanning}

Fixed values for $\gamma_{1}$ and $\gamma_{2}$ would allow the laser beam to be positioned at a desired location in the target plane for the traditional point-by-point scanning measurement. Individual wedges with deviation angles up to $15^{\circ}$ (wedge angle approximately $25^{\circ}$ ) are available, offering up to a $\pm 30^{\circ}$ field of view.

A line-scan could be synthesised from the required series of points but a good approximation to a line scan can be created simply through wedge rotation at the equal speed but opposite directions, as shown in Figure 2(a). The horizontal orientation results when the wedges have the initial orientation shown in Figure 1(a). Advancing the initial angular position of wedge 2 in $45^{\circ}$ increments up to $180^{\circ}$ results in the rotation of the line-scan orientation in increments of half of $45^{\circ}$ until the line-scan attains a vertical orientation. Further increments in the initial angular position of wedge 2 result in further rotation of the line-scan orientation. Note that the "out" and "back" paths in each line deviate from each other and the configuration was chosen deliberately to emphasise this deviation. In Figure 2(a), the deviation is around $\pm 6.6 \mathrm{~mm}$ or $1.5 \%$ of the scan length.

Wedge angle is the primary influence on this deviation, which is also affected, albeit to a much lesser extent, by wedge thickness and separation but which is not affected by the axial distance from wedges to target. Figure 2(b) shows a much improved scan (note the factor of 1000 between horizontal and vertical axes) with the same scan length but maximum deviation of only $\pm 0.25 \mathrm{~mm}$. This decrease, by a factor of approximately 25 is achieved by reducing wedge angle by a factor of approximately 5, with scan length maintained by increasing the axial distance from wedges to target by a factor of 5 . Figure 2(b) shows the complexity in the detailed scan paths. Despite this, the deviation in the "out" and "back" scan paths is now comparable with beam diameter and much smaller than the physical size of a contacting transducer making this approach to line scanning a practical option. 


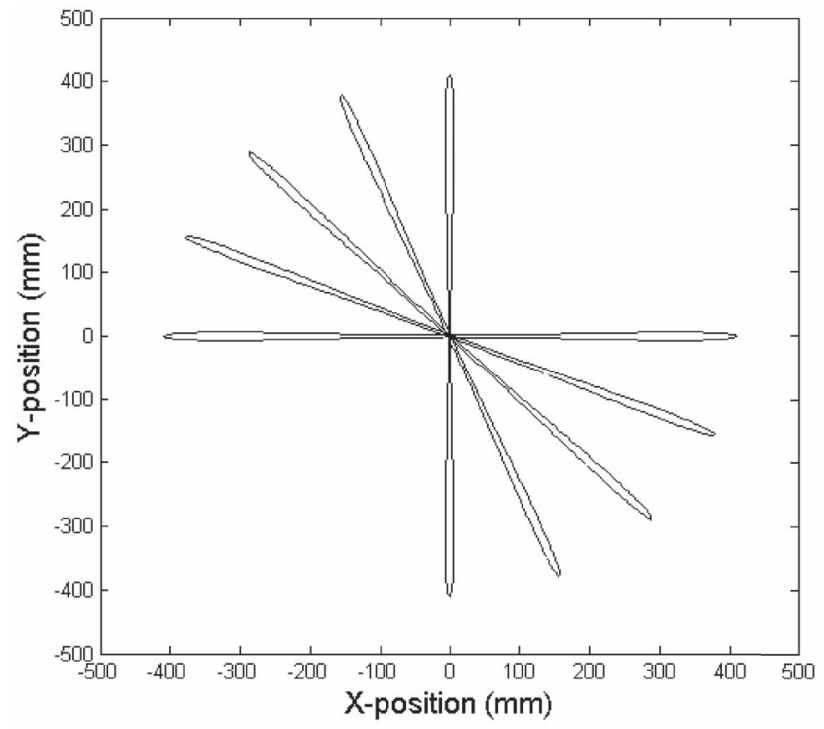

(a)

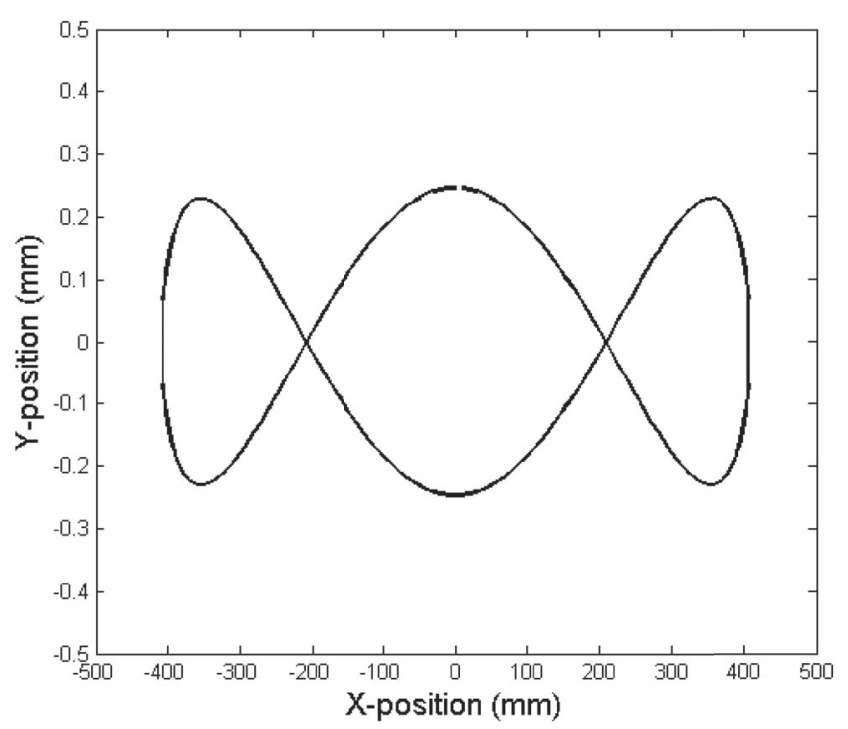

(b)

FIG. 2. Line scans resulting from wedge rotations at equal speed but opposite direction: (a) wedge 2 initial phase stepped in $45^{\circ}$ increments from $0^{\circ}$ to $180^{\circ}$, wedge angles $18^{\circ} 9^{\prime}$, wedge separation $\mathrm{CD}=1 \mathrm{~cm}, \mathrm{z}_{\mathrm{B}}=1.2 \mathrm{~m}$. (Reprinted with permission from S. J. Rothberg and M. Tirabassi, AIP Conf. Proc. 1457, 5 (2012). Copyright 2012 American Institute of Physics. ${ }^{34}$ ) (b) Wedge 2 initial phase $0^{\circ}$, wedge angles $3^{\circ} 53^{\prime}$, wedge separation $\mathrm{CD}=0.2 \mathrm{~cm}$, $\mathrm{z}_{\mathrm{B}}=6 \mathrm{~m}$.

\section{B. Tracking}

If the intention is to scan a circle, such as might be required to track a point on a target rotating at angular speed $\Omega_{T}$, then suitable functions are

$$
\begin{aligned}
& \gamma_{1}=\Omega_{T} t+\phi_{1}, \\
& \gamma_{2}=\Omega_{T} t+\phi_{2},
\end{aligned}
$$

in which the difference between the initial angular positions of the wedges, $\phi_{1}$ and $\phi_{2}$, determines the scan radius.

In assessing the merits of a newly proposed tracking system, the main areas of interest are any deviations between the desired and actual scan paths and three distinct features of the total measured velocity. These features are the effects of misalignments of the optical system in the presence of target rotation, the sensitivity to target out of plane vibration (the intended measurement), and the sensitivity to target inplane vibration (an undesired additional measured velocity). The model set out in this paper is well suited to such an evaluation in MATLAB.

In the absence of misalignments, the scan path of the dual wedge SLDV is effectively a perfect circle and is very accurately centred on the optical axis. To study misalignments, a very large number of misaligned scenarios have been considered. Resulting scans are checked to ensure that scan radius is within $5 \%$ of the intended radius and that the scan centre location is also within $5 \%$ of the scan radius from its intended location. Table I, which compares the performance of the proposed tracking system with those reported previously, ${ }^{29}$ indicates selection of a much smaller number of configurations than the larger number of configurations initially considered, as a result of these checks, for the calculations of measured velocity.

TABLE I. Mean (standard deviation) of rms measured velocities $(\mu \mathrm{m} / \mathrm{s} / \mathrm{rad} / \mathrm{s})$ associated with system configuration and typical misalignments. $50 \mathrm{~mm}$ scan

\begin{tabular}{|c|c|c|c|c|c|c|}
\hline \multirow[b]{2}{*}{ SLDV system } & \multirow{2}{*}{$\begin{array}{l}\text { Misaligned configurations } \\
\text { used/considered }\end{array}$} & & \multicolumn{4}{|c|}{ Order } \\
\hline & & $\mathrm{DC}$ & $0.5 \times$ & $1 \times$ & $1.5 \times$ & $2 \times$ \\
\hline \multirow[t]{2}{*}{ Dual mirror } & No misalignments & No peak & No peak & No peak & No peak & 29.4 \\
\hline & $50621 / 1476225$ & $5.19(4.56)$ & No peak & $284(130)$ & No peak & $29.4(2.02)$ \\
\hline \multirow[t]{2}{*}{ Dove prism } & No misalignments & No peak & 0.170 & 3.85 & $3.01 \times 10^{-2}$ & $7.84 \times 10^{-5}$ \\
\hline & $15155 / 540225$ & $8.72(7.89)$ & $\begin{array}{l}0.219 \\
\left(4.17 \times 10^{-2}\right)\end{array}$ & $221(113)$ & $\begin{array}{l}6.77 \times 10^{-2} \\
\left(2.87 \times 10^{-2}\right)\end{array}$ & $\begin{array}{l}4.55 \times 10^{-4} \\
\left(2.65 \times 10^{-4}\right)\end{array}$ \\
\hline \multirow[t]{2}{*}{ Dual wedges $^{\mathrm{a}}$} & No misalignments & No peak & No peak & No peak & No peak & No peak \\
\hline & $229635 / 1476225$ & $6.00(6.09)$ & No peak & $110(51.7)$ & No peak & $0.130(0.0798)$ \\
\hline
\end{tabular}
radius.

a Simulation parameters: $z_{A}=1.4 \mathrm{~m}, z_{B}=1.2 \mathrm{~m}, z_{D}=1.185 \mathrm{~m},|\rightarrow B C|=|\rightarrow D E|=5 \mathrm{~mm}, \varepsilon_{1}=\varepsilon_{2}=1.5, \psi_{11}=0^{\circ}, \psi_{12}=4^{\circ}, \psi_{21}=4^{\circ}$, and $\psi_{22}=0^{\circ} . \alpha_{L}$ from $-0.1^{\circ}$ to $0.1^{\circ}$ in steps of $0.1^{\circ}, \beta_{L}$ from $-0.4^{\circ}$ to $0.4^{\circ}$ in steps of $0.2^{\circ}, \Delta x_{A}$ from $-10 \mathrm{~mm}$ to $10 \mathrm{~mm}$ in steps of $5 \mathrm{~mm}, \Delta y_{A}$ from $-2 \mathrm{~mm}$ to $2 \mathrm{~mm}$ in steps of $2 \mathrm{~mm}, \alpha_{1}$ and $\alpha_{2}$ from $-0.2^{\circ}$ to $0.2^{\circ}$ in steps of $0.2^{\circ}, \beta_{1}$ and $\beta_{2}$ from $-1^{\circ}$ to $1^{\circ}$ in steps of $1^{\circ}, \Delta x_{B}, \Delta y_{B}, \Delta x_{D}$, and $\Delta y_{D}$ from $-3 \mathrm{~mm}$ to $3 \mathrm{~mm}$ in steps of $3 \mathrm{~mm}$. 


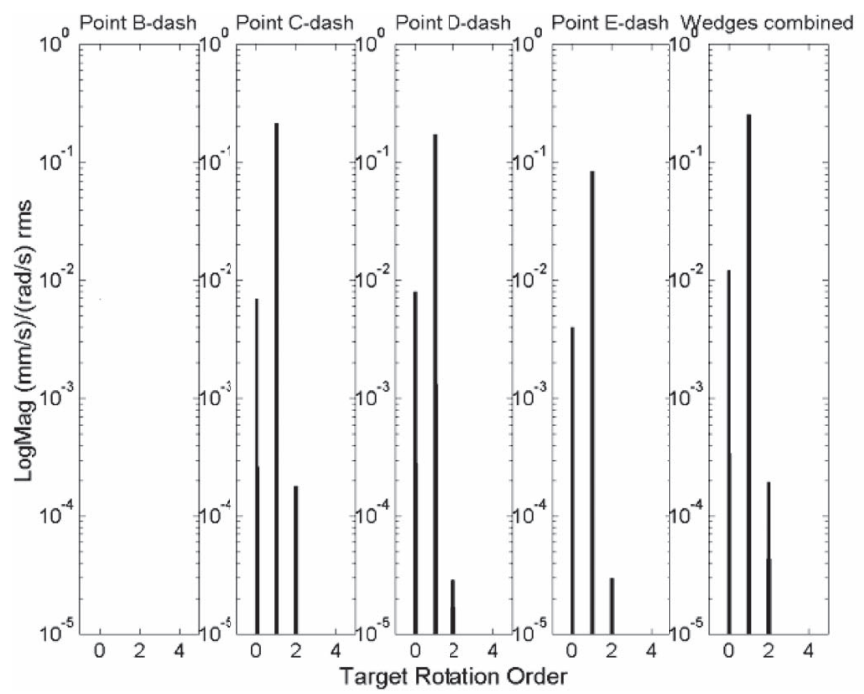

(a)
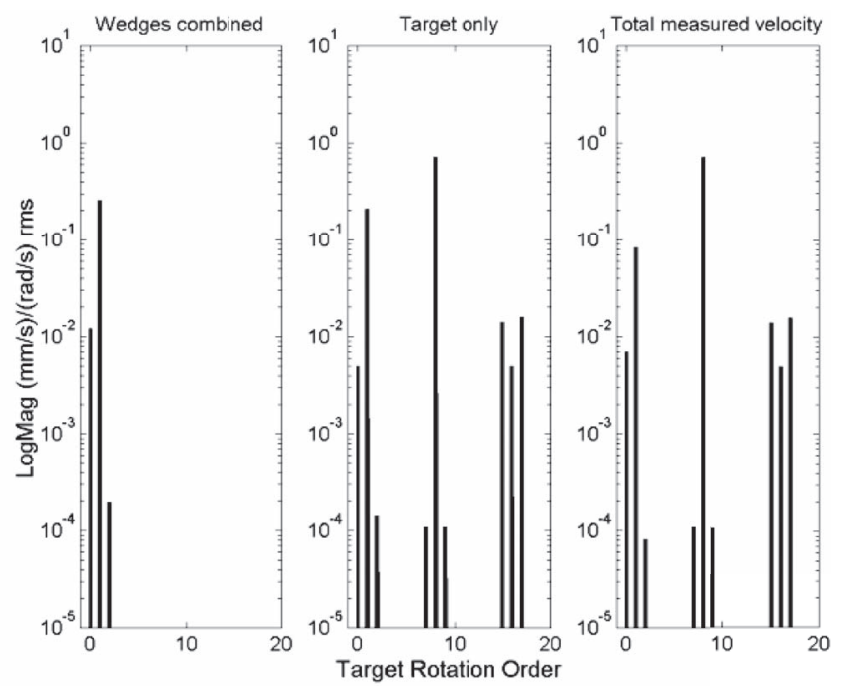

(b)

FIG. 3. Typical velocities in the dual wedge SLDV system in the presence of misalignments $\left(\alpha_{L}=0.1^{\circ}, \beta_{L}=-0.4^{\circ}, \Delta x_{A}=-8 \mathrm{~mm}, \Delta y_{A}=-3 \mathrm{~mm}\right.$, $\left.\alpha_{1}=-0.1^{\circ}, \beta_{1}=0.4^{\circ}, \Delta x_{B}=2 \mathrm{~mm}, \Delta y_{B}=-2 \mathrm{~mm}, \Delta z_{B}=0 \mathrm{~mm}, \alpha_{2}=0.1^{\circ}, \beta_{2}=-0.4^{\circ}, \Delta x_{D}=2 \mathrm{~mm}, \Delta y_{D}=-2 \mathrm{~mm}, \Delta z_{D}=0 \mathrm{~mm}\right)$ with $50 \mathrm{~mm} \mathrm{scan}$ radius and sinusoidal target vibrations $\left(\overrightarrow{V_{f z}}\right.$ of amplitude $10 \mathrm{~mm} / \mathrm{s}$ at $8 \Omega_{\mathrm{T}}$ and $\overrightarrow{V_{O x}}$ of amplitude $10 \mathrm{~mm} / \mathrm{s}$ at $\left.16 \Omega_{\mathrm{T}}\right)$ : (a) the individual and combined effects of the deflection points on the wedges and (b) the combined effect of the wedges, the effect of the target and total measured velocity. Reprinted with permission from S. J. Rothberg and M. Tirabassi, AIP Conf. Proc. 1457, 5 (2012). Copyright 2012 American Institute of Physics. ${ }^{34}$

In the simulations, vibration frequencies are specifically chosen to allow the effects of misalignment, out-of-plane vibration, and in-plane vibration to be distinguished from one another. For the dual wedge SLDV in the presence of the typical misalignments listed, Figure 3(a) shows velocities associated with the deflections at each wedge deflection point while Figure 3(b) shows the combination of velocities from the wedges, from the target, and the total measured velocity. These are calculated in accordance with Eq. (19). With the misalignments chosen, this scan path had a mean radial position of $100.0 \%$ (standard deviation $0.05 \%$ ) and a centre position error of $3.7 \%$, each relative to the intended $50 \mathrm{~mm}$ scan radius. Spectral peaks from DC to $2 \times$ target rotation order show the effect of misalignments on the total measured velocity. Without misalignments, only DC velocities are associated with Doppler shifts at the second wedge and the target and these cancel completely in the total measured velocity. With misalignments, larger components at $\mathrm{DC}$ and $1 \times$, together with a small $2 \times$ component appear, as shown in Figure 3(a). Spectral peaks at the same orders are also associated with Doppler shifts at the target, with useful reduction in the larger components (for this set of misalignments) occurring in the addition of the velocities from the wedges and the target. Consideration of whether Doppler shifts contributing to the total measured velocity originate at optical devices or at the target and consideration of their combination emphasise the comprehensive detail available in these models.

Across a much greater number of misalignments, Table I shows how misalignments affect the low orders in a tracking LDV measurement. The table summarises simulations of a very large number of misaligned configurations for each system. Realistic misalignment values are used at all times (as listed) but the resulting scans are also checked to ensure that scan radius (defined as the mean radial position from the scan centre location) is within $5 \%$ of the intended $50 \mathrm{~mm}$ and that the scan centre location itself is less than $5 \%$ of the scan radius from its intended location. Only scans passing these tests are used for the analysis summarised in Table I, i.e., for the dual wedges, 1476225 misaligned configurations were considered of which 229635 passed the tests set out above and the apparent velocity data presented are based on these.

In the dual wedge system, measurable peaks would be expected at DC and $1 \times$ rotation speed. There appear to be advantages over the dual mirror and Dove prism systems in terms of apparent velocity levels and in the absence of the $2 \times$ component familiar in dual mirror systems and the $0.5 \times$ component characteristic of the Dove prism system. Figure 3(b) shows an out-of-plane flexible vibration at $8 \times$ rotation speed. This characteristic differs little between the three systems considered here with sensitivity of $99.9 \%$ with and without misalignment. Misalignments tend to encourage sidebands but they are of very small amplitude and unlikely to be observable in measured data. Figure 3(b) also shows sensitivity to an in-plane whole body vibration at $16 \times$ rotation speed. Without misalignment, there is no peak at the vibration frequency but sensitivity of approximately $2 \%$ is found at its $\pm \Omega_{\mathrm{T}}$ sidebands. With misalignments, these sidebands are unaffected but a measurable peak at the in-plane vibration frequency itself now appears for all systems with sensitivity around $0.5 \%$.

\section{Synchronised scanning}

The ability to scan while tracking has been demonstrated previously $^{20,21}$ and can be replicated with the dual wedge SLDV using the following rotation angle functions:

$$
\begin{aligned}
& \gamma_{1}=\Omega_{T} t+\phi_{1}-\Delta \gamma \sin m \Omega_{T} t, \\
& \gamma_{2}=\Omega_{T} t+\phi_{2}+\Delta \gamma \sin m \Omega_{T} t .
\end{aligned}
$$




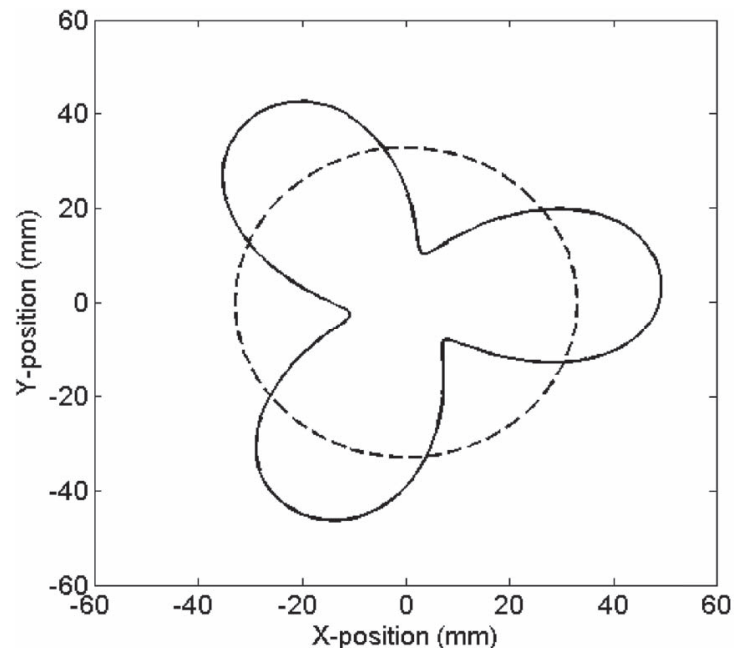

(a)

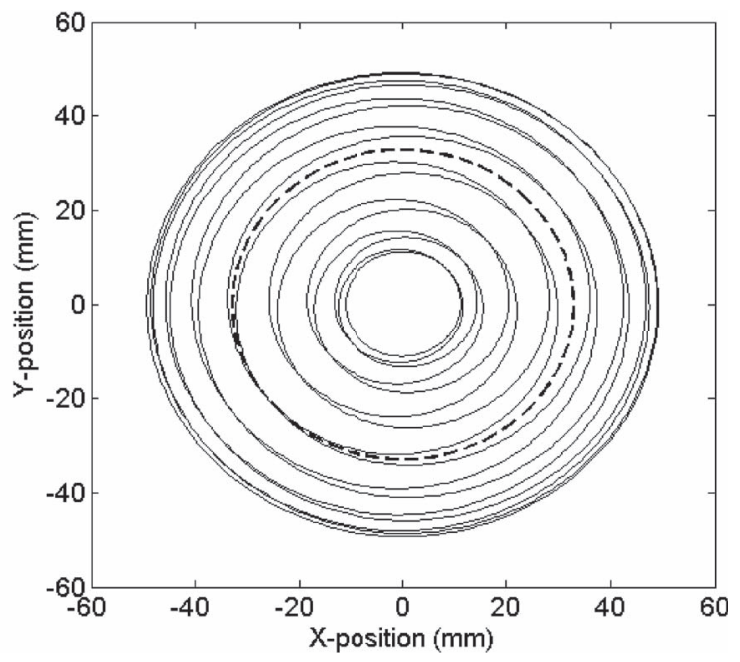

(b)

FIG. 4. Typical synchronised scan paths (solid lines) and corresponding circular scan paths (dashed lines) for the cases where (a) scan frequency higher than rotation frequency $(m=3)$ as in Ref. 20 and (b) scan frequency lower than rotation frequency $(m=1 / 16)$ as in Ref. 21 .

The typical application would be to scan up and down a rotating blade during rotation. In such an application, $\Delta \gamma$ determines the linear extent of the scan while $m$ determines the ratio between rotation frequency and scan frequency. Figure 4 shows two examples together with the circular scan resulting for $\Delta \gamma=0$. In Figure 4(a), the laser beam completes 3 scan cycles in each rotation $(m=3)$ while, in Figure $4(b), 1$ scan cycle is completed over the course of 16 rotation cycles $(m=1 / 16)$.

\section{DUAL WEDGE SLDV: IMPLEMENTING A PRACTICAL SYSTEM}

Figure 5 shows the dual wedges scanning assembly that has been designed and tested for the circular scanning and tracking application in Sec. III B at rotation speeds up to 1800 rpm. The laser beam enters from the left side of the assembly. The wedges can be mounted in either orientation, i.e., with their sloped face as the first or second refraction. In either

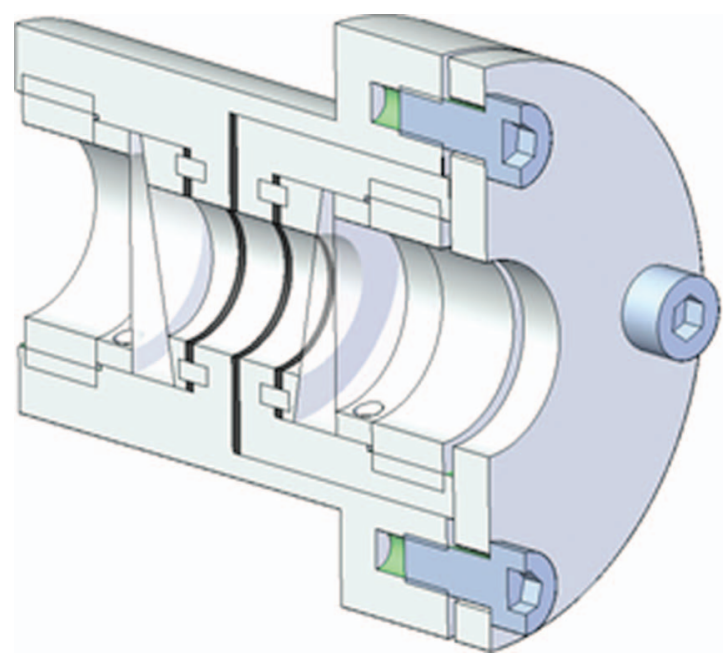

FIG. 5. Dual wedge assembly for circular scanning and tracking. case, the wedge is held in position by a part with complementary geometry against its sloped face and by a part with uniform outer geometry on its perpendicular face. This second part has had material removed by drilling to ensure that each wedge/mount assembly is balanced. The right hand wedge, through which the laser beam exits and passes to the target, is mounted in an assembly that allows adjustment of its angular position relative to the first wedge in order to control the radius of the scanned circle. Figure 6 shows the effect on scan radius (relative to that achieved with a single wedge) of relative angular position for identical wedges. Minimising the wedge separation causes the minimum radius to be closer to zero and the maximum radius to be closer to double.

Initial experimentation has confirmed the ability of the new system to measure vibration while tracking a point on a rotating target. The presence of a dominant component at rotation frequency related to misalignments (see Table I) has also been confirmed and will be explored further here. Exact simulation of real experimental setups is affected by the

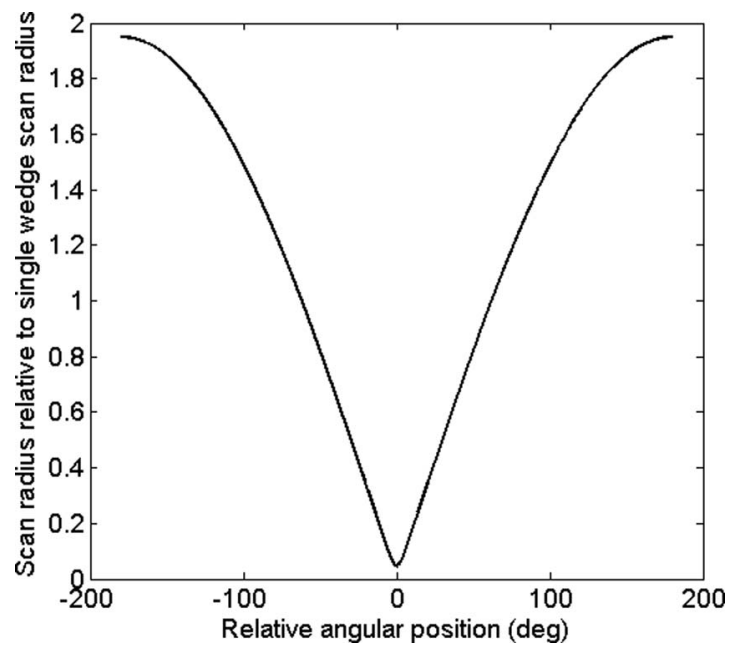

FIG. 6. The effect of relative angular position between identical wedges on scan radius. 


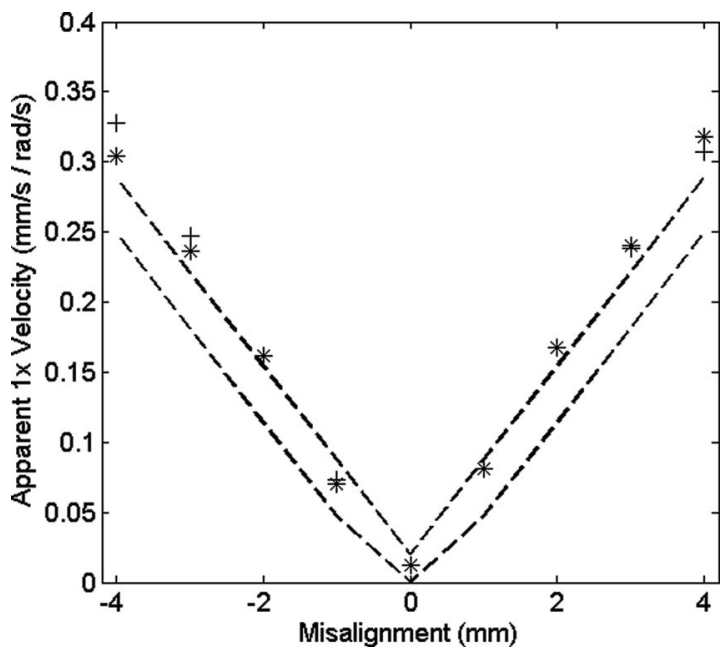

FIG. 7. Theoretical and experimental comparison of apparent velocity with introduction of known translational misalignments of the scanning head in horizontal $(+)$ and vertical $(*)$ directions.

existence of small but inevitable misalignments in the experimental arrangement that cannot be quantified directly. Based on the specific experimental arrangement, 59049 misaligned but plausible configurations were considered in which two angular and two translational misalignments were included for the laser itself and the two wedges (12 misalignments in all). The simulated data shown in Figure 7 are based on consideration of 22911 misaligned configurations (out of the full set of 59049); those chosen all resulted in an apparent velocity close to the measured value for zero added translational misalignment. The dashed lines show the range of apparent velocities predicted from the chosen configurations with the expectation that the measured velocities should fall in this range. Given the uncertainty associated with the unknown misalignments, this level of agreement is regarded very positively.

\section{CONCLUSIONS}

The beam path, scan path, and measured velocity associated with a proposed LDV scanning head based on rotating optical wedges have been comprehensively and successfully modelled. Simulations performed suggest that the dual wedge SLDV can be a versatile alternative to the dual mirror SLDV and other reported SLDV systems, with advantages including higher frequency operation and lower apparent velocities at key rotation orders. Experimental data have confirmed the viability of the proposed system and the validity of the mathematical model presented.

\section{ACKNOWLEDGMENTS}

The authors gratefully acknowledge financial support from the Engineering and Physical Sciences Research Council and the support of Andrew Snart in the design of the scanning head.

\section{APPENDIX: ROTATION MATRICES}

Rotation matrices are as follows:

$$
\begin{aligned}
& {[x, \alpha]=\left[\begin{array}{ccc}
1 & 0 & 0 \\
0 & \cos \alpha & -\sin \alpha \\
0 & \sin \alpha & \cos \alpha
\end{array}\right],} \\
& {[y, \beta]=\left[\begin{array}{ccc}
\cos \beta & 0 & \sin \beta \\
0 & 1 & 0 \\
-\sin \beta & 0 & \cos \beta
\end{array}\right],} \\
& {[z, \gamma]=\left[\begin{array}{ccc}
\cos \gamma & -\sin \gamma & 0 \\
\sin \gamma & \cos \gamma & 0 \\
0 & 0 & 1
\end{array}\right] .}
\end{aligned}
$$

${ }^{1}$ B. Stoffregen and A. Felske, "Scanning laser Doppler vibration analysis system," SAE Technical Paper 850327 (1985).

${ }^{2}$ B. Junge, "Experiences with scanning laser vibrometry in automotive industries," Proc. SPIE 2358, 377-382 (1994).

${ }^{3}$ X. D. Zeng, A. L. Wicks, and T. E. Allen, "Pose estimation of a scanning laser Doppler vibrometer with applications to the automotive industry," Opt. Eng. 37(5), 1442-1447 (1998).

${ }^{4}$ M. A. Beeck and W. Hentschel, "Laser metrology-a diagnostic tool in automotive development processes," Opt. Lasers Eng. 34(2), 101-120 (2000).

${ }^{5}$ J. Hancox, B. C. Staples, and R. J. Parker, "The application of scanning laser Doppler vibrometry in aero-engine development," Proc. IMechE - J. Aerosp. Eng. 209, 35-42 (1995).

${ }^{6}$ Polytec, "Structure-borne noise measurement with a robot-controlled 3-D scanning laser vibrometer," InFocus 1, 5-7 (2008); see http://www. polytec.com/no_cache/int/news/infocus-magazine/?cid=3280\&did=3622\& sechash $=5$ bca964d (June 2013).

${ }^{7}$ A. B. Stanbridge and D. J. Ewins, "Measurement of translational and angular vibration using a scanning laser Doppler vibrometer," Shock Vib. 3(2), 141-152 (1996).

${ }^{8}$ P. Sriram, J. I. Craig, and S. Hanagud, "A scanning laser Doppler vibrometer for modal testing," Int. J. Anal. Exp. Modal Anal. 5(3), 155-167 (1990).

${ }^{9}$ A. B. Stanbridge and D. J. Ewins, "Modal testing using a scanning laser Doppler vibrometer," Mech. Syst. Signal Process. 13(2), 255-270 (1999).

${ }^{10}$ S. Vanlanduit, P. Guillaume, and J. Schoukens, "Broadband vibration measurements using a continuously scanning laser vibrometer," Meas. Sci. Technol. 13(10), 1574-1582 (2002).

${ }^{11}$ J. P. La, J. Choi, S. Y. Wang, K. Kim, and K. Park, "Continuous scanning laser Doppler vibrometer for mode shape analysis," Opt. Eng. 42(3), 730737 (2003).

${ }^{12}$ A. B. Stanbridge, M. Martarelli, and D. J. Ewins, "Measuring area vibration mode shapes with a continuous-scan LDV," Measurement 35(2), 181-189 (2004).

${ }^{13}$ M. S. Allen and M. W. Sracic, "A new method for processing impact excited continuous-scan laser Doppler vibrometer measurements," Mech. Syst. Signal Process. 24(3), 721-735 (2010).

${ }^{14}$ P. Giuliani, D. DiMaio, C. W. Schwingshackl, M. Martarelli, and D. J. Ewins, "Six degrees of freedom measurement with continuous scanning laser Doppler vibrometer," Mech. Syst. Signal Process. 38, 367-383 (2013).

${ }^{15}$ P. Castellini and N. Paone, "Development of the tracking laser vibrometer: performance and uncertainty analysis," Rev. Sci. Instrum. 71(12), 46394647 (2000)

${ }^{16} \mathrm{~B}$. J. Halkon and S. J. Rothberg, "Vibration measurements using continuous scanning laser vibrometry: advanced aspects in rotor applications," Mech. Syst. Signal Process. 20(6), 1286-1299 (2006).

${ }^{17}$ S. Zucca, D. DiMaio, and D. J. Ewins, "Measuring the performance of underplatform dampers for turbine blades by rotating laser Doppler vibrometer," Mech. Syst. Signal Process. 32, 269-281 (2012).

${ }^{18} \mathrm{P}$. Castellini and R. Montanini, "Automotive components vibration measurements by tracking laser Doppler vibrometry: advances in signal processing," Meas. Sci. Technol. 13, 1266-1279 (2002).

${ }^{19}$ H. Dietzhausen, K. Bendel, and N. Scelles, "Tracking scanning laser Doppler vibrometers: extending laser vibrometry to arbitrarily moving objects," in Proceedings of the IMAC XXI: A Conference and Exposition on Structural Dynamics, Kissimmee, Florida, 2003. 
${ }^{20}$ B. J. Halkon and S. J. Rothberg, "Synchronised-scanning laser vibrometry," Proc. SPIE 5503, 260-271 (2004).

${ }^{21}$ D. Di Maio and D. J. Ewins, "CAISER MYMESIS: a new software platform for virtual and actual vibration testing on rotating structures using a continuously scanning LDV technique," Proc. SPIE 6345, 63450Q (2006).

${ }^{22}$ R. A. Lomenzo, A. J. Barker, and A. L. Wicks, "Laser vibrometry system for rotating bladed disks," in Proceedings of the 17th International Modal Analysis Conference (SEM, Kissimmee, FL, 1999), Vol. 3727, pp. 277282.

${ }^{23}$ I. A. Sever, A. B. Stanbridge, and D. J. Ewins, "Turbomachinery blade vibration measurements with tracking LDV under rotation," Proc. SPIE 6345, 63450L (2006).

${ }^{24}$ D. Di Maio and D. J. Ewins, "Applications of continuous tracking SLDV measurement methods to axially symmetric rotating structures using different excitation methods," Mech. Syst. Signal Process. 24(8), 30133036 (2010)

${ }^{25}$ S. Boedecker, A. Drabenstadt, L. Heller, A. Kraft, A. Leonhardt, C. Pape, S. Ristau, E. Reithmeier, and C. Rembe, "Optical derotator for scanning vibrometer measurements on rotating objects," Proc. SPIE 6345, 63450M (2006).

${ }^{26}$ Polytec Application Note, "Surface vibration measurement on rotating components: Non-contact deflection shape analysis on rotating compo- nents using the PSV-A-440 optical derotator," see http://www.polytec.com/ eu/products/vibration-sensors/scanning-vibrometers/psv-a-440-opticalderotator/ (June 2013).

${ }^{27}$ F. A. Rosell, "Prism scanner," J. Opt. Soc. Am. 50(6), 521-526 (1960).

${ }^{28}$ G. F. Marshall, "Risley prism scan patterns," Proc. SPIE 3787, 74-86 (1999).

${ }^{29}$ S. J. Rothberg and M. Tirabassi, "A universal framework for modelling measured velocity in laser vibrometry with applications," Mech. Syst. Signal Process. 26, 141-166 (2012).

${ }^{30}$ B. M. Watrasiewicz and M. J. Rudd, Laser Doppler Measurements (Butterworths, London, Boston, 1976).

${ }^{31}$ J. R. Bell and S. J. Rothberg, "Laser vibrometers and contacting transducers, target rotation and six degree-of-freedom vibration: what do we really measure?," J. Sound Vib. 237(2), 245-261 (2000).

${ }^{32}$ B. J. Halkon and S. J. Rothberg, "Vibration measurements using continuous scanning laser Doppler vibrometry: theoretical velocity sensitivity analysis with applications," Meas. Sci. Technol. 14, 382-393 (2003).

${ }^{33}$ An Introduction to Ray Tracing, edited by A. S. Glassner (Academic Press, London, 1989).

${ }^{34}$ S. J. Rothberg and M. Tirabassi, "Are rotating wedges a feasible alternative to dual mirrors for scanning and tracking LDV?," AIP Conf. Proc. 1457, 5 (2012). 